\title{
Heterozygous Mylk3 Knockout Mice Partially Recapitulate Human DCM With Heterozygous MYLK3 Mutations
}

\author{
Carson L. Tougas, Tabor Grindrod, Lawrence X. Cai, Fariz F. Alkassis and \\ Hideko Kasahara*
}

Department of Physiology and Functional Genomics, College of Medicine, University of Florida, Gainesville, FL, United States

\section{OPEN ACCESS}

Edited by:

Julian Stelzer,

Case Western Reserve University,

United States

Reviewed by:

Danuta Szczesna-Cordary,

University of Miami, United States

Tom Burghardt,

Mayo Clinic, United States

*Correspondence: Hideko Kasahara

hkasahar@ufl.edu

Specialty section:

This article was submitted to Striated Muscle Physiology, a section of the journal

Frontiers in Physiology

Received: 08 February 2019

Accepted: 16 May 2019

Published: 06 June 2019

Citation:

Tougas CL, Grindrod T, Cai LX, Alkassis FF and Kasahara H (2019) Heterozygous Mylk3 Knockout Mice Partially Recapitulate Human DCM

With Heterozygous MYLK3

Mutations. Front. Physiol. 10:696. doi: 10.3389/fphys.2019.00696
Backgrounds: Recent studies identified heterozygous variants in MYLK3 gene that encodes cardiac myosin light chain kinase (CMLCK) are related to familial dilated cardiomyopathy $(\mathrm{DCM})$ for the first time. Autosomal dominant traits suggest that pathogenesis of DCM could be related to heterozygous MYLK3 loss-of-function variants (haploinsufficiency). We previously generated and examined homozygous Mylk3 knockout mice that lead to heart failure. It had yet to be examined whether heterozygous Mylk3 knockout mice represent a DCM-like phenotype.

Methods and Results: Heterozygous knockout (Mylk3 $3^{\text {wild} /-}$ ) mice were examined regarding cardiac function, heart histology and expression of CMLCK protein and mRNA relative to age-matched wild-type controls (Mylk3 $3^{\text {wild/wild }}$ ). At 4 months of age, cardiac contractility in heterozygous knockout mice was reduced with percent fractional shortening of $23.3 \pm 1.2 \%$ compared to $30.1 \pm 1.8 \%$ in control (Mylk3 $3^{\text {wild/ }}-$ vs. Mylk3wild/wild, $n=9$ each). In 4-month-old heterozygous knockout hearts, expression of cMLCK mRNA was expectedly reduced by almost half, however, protein expression was reduced by approximately $75 \%$ relative to the control wildtype (Mylk3 $3^{\text {wild/- }}$ vs. Mylk3wild/wild, $n=9$ each). Isolated ventricular cardiomyocytes from heterozygous knockout mice were larger with increase of short-axis length relative to the cardiomyocytes from control mice. However, increase of heart failure markers as well as interstitial fibrosis were not evident in heterozygous knockout mice compared to controls.

Conclusion: Heterozygous Mylk3 knockout mice show mild reduction of cardiac contractility by 4 months of age, and proteins reduced by approximately $75 \%$ relative to the control wild-type mice. These mice partly resemble human with the heterozygous MYLK3 mutation, but the reduction in cardiac contractility was milder.

Keywords: kinase, heart, genetic dilated cardiomyopathy, heterozygous knockout, animal model of human disease

\section{INTRODUCTION}

The dynamics of cardiac contraction and relaxation are fundamentally related to actin-myosin interactions. Myosin is a hexamer composed of two heavy chains and two pairs of light chains (MLC1 and MLC2). Ventricular MLC2 (MLC2v) is bound to the neck of the heavy chain, which acts as a lever arm to generate movement, and MLC2v phosphorylation has been shown to potentiate 
the rate and force of cardiac contraction (Sanbe et al., 1999; Davis et al., 2001; Moss and Fitzsimons, 2006; Stelzer et al., 2006; Scruggs and Solaro, 2011). The predominant kinase for MLC2v is cardiac MLCK (cMLCK) encoded by Mylk3 in mice and MYLK3 in humans. Reduced phosphorylation of MLC2 $v$ has been implicated in human heart disease where phosphorylation was reduced to $\sim 18 \%$ of total MLC $2 \mathrm{v}$ in failing hearts from $\sim 30$ to $40 \%$ in healthy hearts (Morano, 1999; van der Velden et al., 2003). In addition, recent studies showed that heterozygous human mutations in MYLK3 gene could be related to familial dilated cardiomyopathy (DCM) (Tobita et al., 2017; Hodatsu et al., 2019).

Haploinsufficiency due to loss-of-function mutations has been documented as a pathological mechanism for a number of human diseases (Marston et al., 2012; Rocha et al., 2016; Bartha et al., 2018). The autosomal dominant nature of MYLK3related DCM suggests that heterozygous loss-of-function may be responsible for pathogenesis. Indeed, homozygous Mylk3 knockout $\left(M y l k 3^{-/-}\right)$hearts were moderately enlarged with increased heart weight at 3 and 6 months of age in sedentary conditions relative to age- and sex-matched (female) control wild-type litters. MLC2v phosphorylation was below the level of detection. Cardiac contraction assessed using magnetic resonance imaging and echocardiography demonstrated a reduction in ejection fraction and increases in the volume of the left ventricular cavity both at end-systole and enddiastole. However, these mice did not show typical heart failure phenotypes, such as an increase in interstitial fibrosis or fetal gene re-expression (Warren et al., 2012). Isolated ventricular cardiomyocytes from adult $M y l k 3^{-/-}$mice were larger than cardiomyocytes from control mice and displayed reductions in contractility and speed of relaxation with no changes in amplitude of the intracellular $\mathrm{Ca}^{2+}$ transient. In contrast to a moderate reduction in contractility under basal conditions, 3 months of transverse aortic constriction resulted in profound heart failure and a decreased survival rate in these mice.

Heterozygous $M y l k 3$ knockout $\left(M y l k 3^{\text {wild/- }}\right.$ ) knockout mice remain unexplored as a human DCM model. The aim of this study is to assess the phenotypic similarities and differences between heterozygous MYLK3 variants in DCM patients versus heterozygous Mylk3 knockout mice. Heterozygous Mylk3 knockout mice demonstrate mild heart failure with markedly reduced expression of cMLCK proteins discordant with mRNA level.

\section{MATERIALS AND METHODS}

\section{Mouse Model}

Germline $M y l k 3^{\text {wild } /-}$ mice were generated as reported in our previous study (Warren et al., 2012), followed by backcrossing with C57BL/6 wild-type mice over six generations. Age- and sex-matched (female) wild-type mice were used as controls. All animal experiments were performed with approval from the University of Florida Institutional Animal Care and Use Committee, which conforms to NIH guidelines.

\section{Echocardiogram and Left Ventricular Pressure Volume (LVPV) Measurement}

Echocardiography of the hearts was performed as described previously (Warren et al., 2012). Briefly, mice were anesthetized with 1.5 to $2 \%$ isoflurane to maintain heart rate around 500 bpm during imaging using Vevo770 (Visual Sonics). LVPV measurements using a PVR1045 catheter (Millar, Houston, TX, United States) were performed under intubation and ventilation with 1 to $2 \%$ isoflurane to maintain heart rate around $450 \mathrm{bpm}$ using standard methods (Pacher et al., 2008) and were analyzed by Lab Chart 8 (AD Instruments, Colorado Springs, CO) followed by the conversion of relative volume unit (RVU) to units of volume using the cuvette calibration.

\section{Measurements of Cardiomyocyte Cell Size, Simultaneous Measurements of Cell Shortening and Intracellular Free Calcium}

Isolated adult cardiomyocytes attached to glass coverslips were imaged under a microscope and digitized for measurements of cell surface area. Rod-shaped cardiomyocytes with clear cross-striations, staircase ends and surface membranes free from blebs were utilized for simultaneous measurements of cell shortening and intracellular free calcium (IonOptix, Westwood, MA, United States), performed as described previously (Takeda et al., 2009).

\section{Western Blotting, Immunostaining and Histological Analyses}

Paraffin-embedded tissue sectioning of $5 \mu \mathrm{m}$ thickness was used for Picro Sirius Red staining and immunostaining as described previously (Warren et al., 2012). Western blot analyses and immunostaining were performed with the following antibodies: cMLCK (Chan et al., 2008), phospho-MLC2v (gift from Dr. N. Epstein, NIH) (Davis et al., 2001), MLC2 (F109.3E1, ALX-BC-1150-S-L005, Enzo Life Science, Farmingdale, NY, United States), $\alpha$-actinin2 (mouse monoclonal A7811 Sigma), myomesin (mouse monoclonal, mMAC myomesin B4, Developmental Studies Hybridoma Bank), and GAPDH (MAB374, MilliporeSigma, Burlington, MA, United States). Fluorescent staining was performed side by side, followed by imaging with the same exposure time below the level of saturation using a ZEISS Axiovert200M (Carl Zeiss AG, Oberkochen, Germany).

\section{Administration of Proteasome Inhibitors}

A combination of Lactacystin $(10 \mu \mathrm{M})$ and MG132 $(5 \mu \mathrm{M})$ (LifeSensors, Malvern, PA, United States) was added on the day of isolation and incubated for $48 \mathrm{~h}$. Cardiomyocytes were scraped, and used for Western blotting.

\section{Real-Time RT-PCR}

Real-time RT-PCR was performed using TaqMan Gene Expression Assays (Applied Biosystems, Foster City, CA, United States): cardiac MLCK Mm00615292, atrial natriuretic 
factor (ANF) Mm01255748, and brain natriuretic peptide (BNP) Mm00435304, followed by normalization to $\beta$-actin expression (No. 4352933E). Duplicated experiments were averaged.

\section{Statistical Analyses}

Data presented are expressed as mean values \pm S.E.M. Results were compared using a student's $T$-test or ANOVA followed by Fisher's post hoc test (SPSS ver. 25). $P<0.05$ was considered significant.

\section{RESULTS}

\section{Heart Enlargement and Reduction of Contractility in Heterozygous Mylk3 Knockout Mice}

By mating heterozygous Mylk3 knockout mice (Mylk ${ }^{\text {wild/- }}$ ), we generated and analyzed age- and sex-matched (female) Mylk3 $3^{\text {wild/ - }}$ relative to the control wild-type litters (Mylk3 $3^{\text {wild/wild }}$ (Figure 1A). There was no change in the heart weight/body weight $(\mathrm{HW} / \mathrm{BW})$ ratio between the two groups at 3 weeks of age ( $6.45 \pm 0.16$ in control vs. $6.49 \pm 0.20 \mathrm{mg} / \mathrm{g}$ in heterozygous knockout mice, $n=5$ and 7 , respectively, $P=0.88$ ). At 4 months of age, while not significant $(P=0.133)$, HW/BW was increased in heterozygous knockout relative to control mice $(4.81 \pm 0.08$ vs. $5.34 \pm 0.32 \mathrm{mg} / \mathrm{g}, n=9$ each) (Figure 1B). Hereafter, we examined 4-month-old mice.

Cardiac contractility examined using echocardiogram demonstrated a significant reduction in contractility and an increase in the LV chamber dimensions both at diastole and systole in heterozygous knockout relative to the controls (\%fractional shortening, $30.1 \pm 1.8$ in control vs. $23.3 \pm 1.2 \%$ in heterozygous knockout mice; end-diastolic dimension, $3.76 \pm 0.14$ vs. $4.30 \pm 0.12 \mathrm{~mm}$; end-systolic dimension, $2.65 \pm 0.15$ vs. $3.30 \pm 0.12 \mathrm{~mm}, n=9$ each, respectively, $P<0.05$; Figures 1C,D and Table 1). Hemodynamic measurements also showed dilation of the LV, in particular, at end-systole, a decrease in end-systolic pressure, and a decrease in LV contractility (a decrease of preload recruitable stroke work, PRSW and end-systolic elastance, Ees) in heterozygous knockout relative to the age- and sex-matched control wild-type mice (Figures 1E,F and Table 2). However, diastolic function was not significantly impaired as represented by the end-diastolic pressure volume relation slope and relaxation time constant (tau).

Histologically, we did not find interstitial fibrosis, which is often seen in the failed heart, in either of two groups using Picro Sirus Red staining (Figure 2A, $n=3$ each). Of note, we did not see interstitial fibrosis in the homozygous knockout mice despite the higher degree of cardiac dysfunction.

\section{Cardiomyocyte Enlargement and Reduction in Contractility in Heterozygous Mylk3 Knockout Mice}

Isolated ventricular cardiomyocytes from 4-month-old heterozygous knockout mice were larger in cell surface area accompanied by increased short-axis length and an increased ratio of short axis relative to long axis compared to those isolated from control mice (cell area, 2,300 \pm 50 in control vs. $2,500 \pm 57 \mu \mathrm{m}^{2}$ in heterozygous knockout; short axis, $21.5 \pm 0.4$ vs. $23.9 \pm 0.4 \mu \mathrm{m}$; short/long axis ratio $0.22 \pm 0.1$ vs. $0.25 \pm 0.01$, $n=371$ and 351, respectively, $P<0.05$; Figure 2B). These cardiomyocytes displayed reductions in contractility and speed of contraction $(+\mathrm{dL} / \mathrm{dt})$ without significant changes in amplitude of the intracellular $\mathrm{Ca}^{2+}$ transient (\%fractional shortening, $3.31 \pm 0.17$ in control vs. $2.70 \pm 0.2 \%$ in heterozygous knockout; $+\mathrm{dL} / \mathrm{dt}, 1.33 \pm 0.06$ vs. $1.11 \pm 0.07 \mu \mathrm{m} / \mathrm{sec}, n=171$ and 158 , respectively, $P<0.05$; Figure 2C).

\section{cMLCK's mRNA and Protein Expression in Heterozygous Mylk3 Knockout Mice}

At 4 months of age, cMLCK protein expression was reduced by more than half (approximately by $75 \%$ ) and MLC2v phosphorylation was reduced by half in heterozygous knockout hearts relative to controls (fold difference cMLCK/GAPDH, $1.00 \pm 0.16$ in control vs. $0.26 \pm 0.09$ in heterozygous knockout; fold difference pMLC2/total MLC2, $1.00 \pm 0.17$ vs. $0.47 \pm 0.06$, $n=9$ each, $P<0.05$; Figures 3 A,B). Of note, we have previously validated a specificity of phospho-specific MLC2 antibody using 2D-gel followed by Western blotting (Warren et al., 2012).

cMLCK mRNA relative to $B$-actin was reduced approximately by half in heterozygous knockout hearts relative to controls (fold difference cMLCK/ $/$-actin, $1.00 \pm 0.05$ in control vs. $0.51 \pm 0.03$ in heterozygous knockout, $n=9$ each, experiment duplicated and averaged, $P<0.05$; Figure 3C). Expression of mRNA for ANF and BNP relative to $\mathrm{B}$-actin was not significantly different between the two groups (Figure 3C), which is consistent with the lack of increase in heart failure markers present in homozygous sedentary knockout mice (Warren et al., 2012). Despite skeletal and smooth muscle MLCK and Dapk3/ZIPK having been shown to phosphorylate MLC2 (Herring et al., 2000; Davis et al., 2001; Chang et al., 2010), there were no compensatory increases of mRNA of these kinases in heterozygous knockout hearts (Figure 3C). This finding was consistent with the homozygous inducible and germline Mylk3 knockout mice, where cMLCK and MLC2 phosphorylation was markedly reduced or absent (Massengill et al., 2016).

\section{Reduction of cMLCK Proteins in Heterozygous Knockout Mice}

We previously showed that the ubiquitin-proteasome system (UPS) is partly responsible for degrading cMLCK proteins in wild-type hearts and neonatal cardiomyocytes (Warren et al., 2012). Using isolated adult cardiomyocytes, we compared the involvement of UPS-dependent protein degradation between heterozygous knockout relative to control. In control cardiomyocytes, unexpectedly, an expression of cMLCK protein was markedly decreased after $48 \mathrm{~h}$ of culture (Figures 4A,B). However, reduction of cMLCK proteins was partly blocked by UPS inhibitors (combination of lactacystin and MG132) as expected. In contrast, the expression of cMLCK proteins in heterozygous knockout cardiomyocytes was lower compared to those from wild-type 


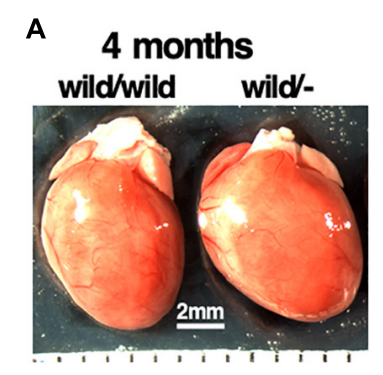

\section{B HW/BW}
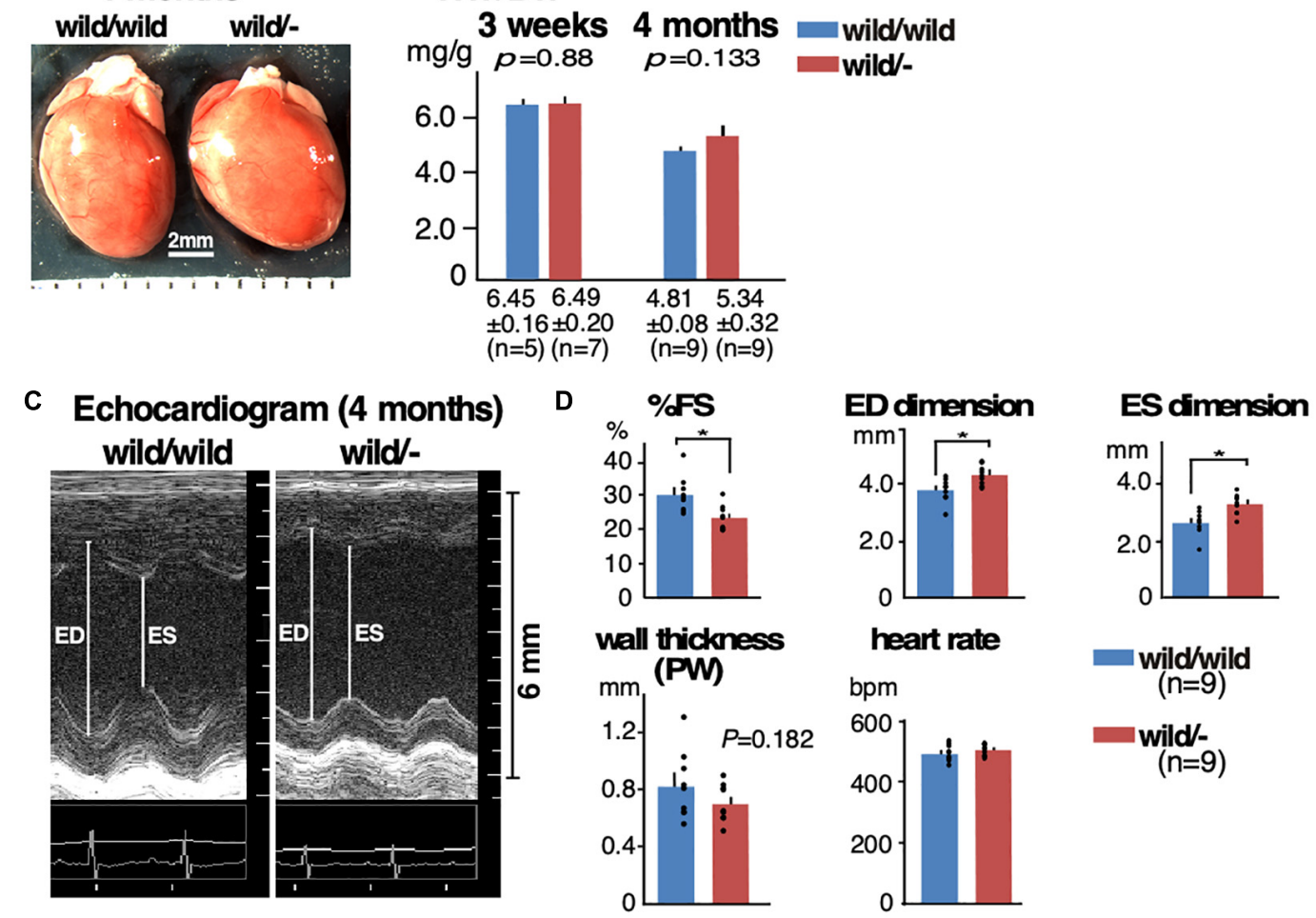

E

$$
\text { LVPV (4 months) }
$$
$\mathrm{mmHg}$ wild/wild
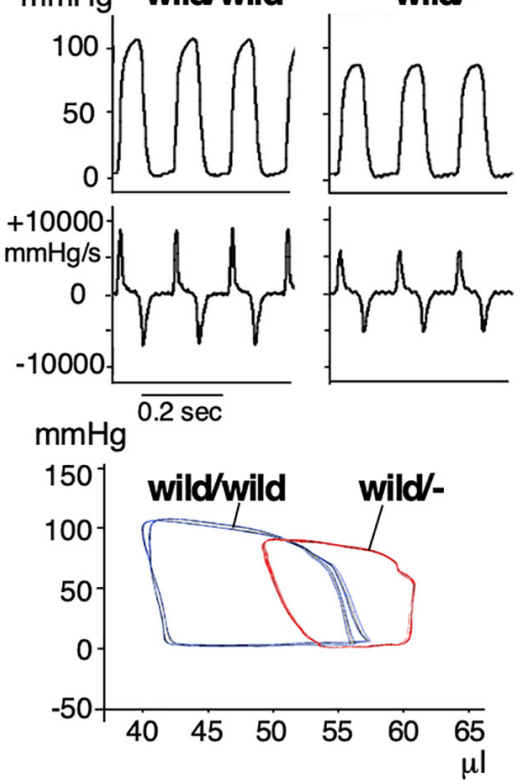

$\mathbf{F}$

$$
\text { wild/wild }(n=7) \quad \text { wild/- }(n=6)
$$

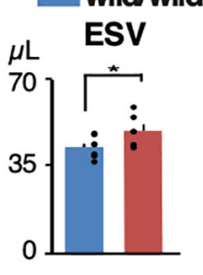

dP/dt max
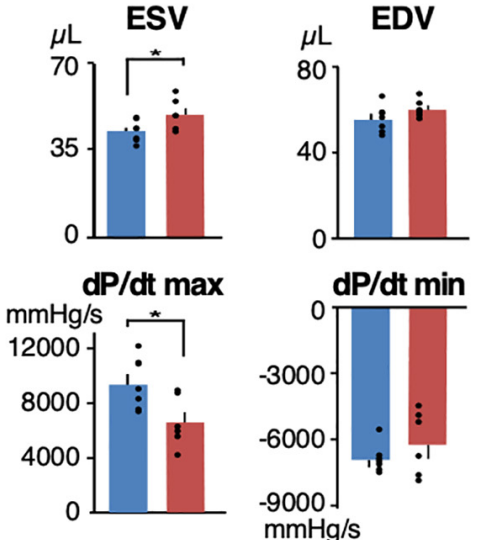

EDPVR slope
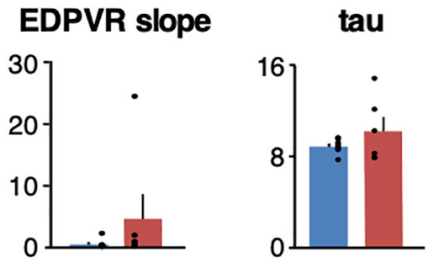
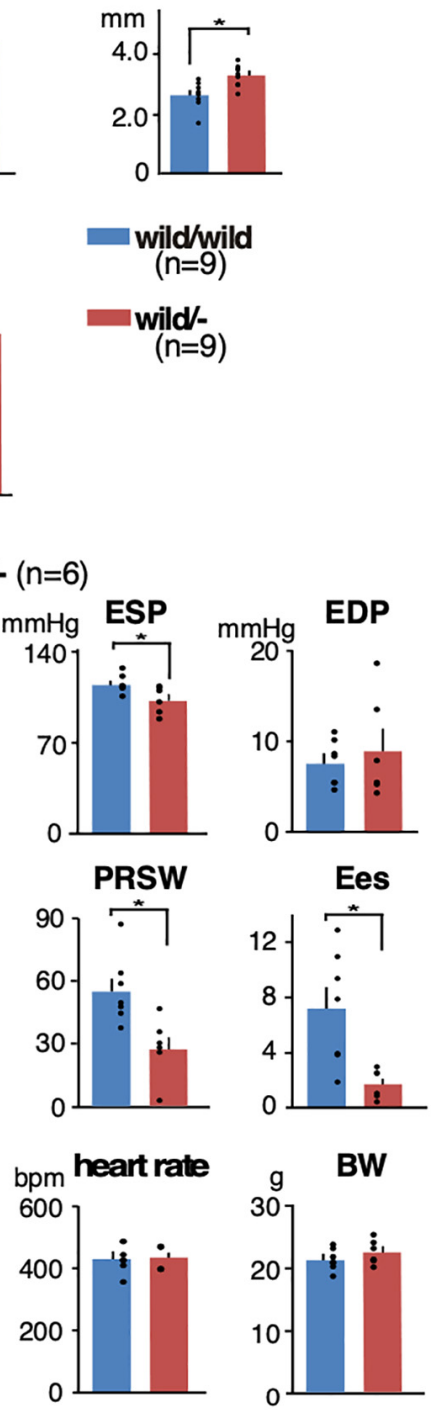

FIGURE 1 | Mild heart enlargement and reduced contractile function in heterozygous Mylk3 knockout mice. (A) By mating Mylk3 wild/- mice, Mylk3 wild/wild, Mylk3 $3^{\text {wild } /-}$, and Mylk3 $^{-/-}$were generated. Representative hearts dissected from three groups of mice at 4 months of age. Bar $=2 \mathrm{~mm}$. (B) HW/BW (mg/g) at 3 weeks and 4 months of age. (C,D) Representative images of M-mode ultrasound and echocardiographic indices of Mylk3wild/wild and Mylk3wild/- mice at

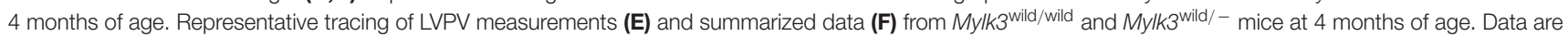
expressed as mean \pm SEM, with or without individual data that are presented by black circles. $P$ values are indicated in panel B, or * indicates $P<0.05$. HW/BW, heart weight/body weight ratio; ED, end-diastolic; ES, end-systolic; \%FS, \% left ventricular fractional shortening; PW, posterior wall; ESV, end-systolic volume; EDV, end-diastolic volume; ESP, end-systolic pressure; EDP, end-diastolic pressure; dP/dt max, peak rate of pressure rise; dP/dt min, peak rate of pressure decline; PRSW, preload recruitable stroke work; Ees, end-systolic elastance; EDPVR slope, end-diastolic PV relation slope; Tau, relaxation time constant. 
TABLE 1 | Summary of echocardiographic indices of Mylk3wild/wild and Mylk3wild/- mice at 4 months of age.

\begin{tabular}{lccc}
\hline & WW $(\boldsymbol{n}=\mathbf{9})$ & W/- $(\boldsymbol{n}=\mathbf{9})$ & $\boldsymbol{P}$ value \\
\hline \%FS & $30.1 \pm 1.8$ & $23.3 \pm 1.2$ & $0.006^{*}$ \\
ED dimension (mm) & $3.76 \pm 0.14$ & $4.30 \pm 0.12$ & $0.009^{*}$ \\
ES dimension (mm) & $2.65 \pm 0.15$ & $3.30 \pm 0.12$ & $0.003^{*}$ \\
Wall thickness (PW) & $0.83 \pm 0.07$ & $0.70 \pm 0.04$ & 0.182 \\
Heart rate (bpm) & $494 \pm 9$ & $503 \pm 6$ & 0.417 \\
\hline
\end{tabular}

ED, end-diastolic; ES, end-systolic; \%FS, \% left ventricular fractional shortening; $P W$, posterior wall. $P, P$ value. ${ }^{*} P<0.05$.

TABLE 2 | Summary of echocardiographic indices of Mylk3 $3^{\text {wild/wild }}$ and Mylk3 $3^{\text {wild } /-~ m i c e ~ a t ~} 4$ months of age.

\begin{tabular}{lccl}
\hline & WW $(\boldsymbol{n}=\mathbf{7})$ & W/- $(\boldsymbol{n}=\mathbf{6})$ & $\boldsymbol{P}$ value \\
\hline ESV $(\mu \mathrm{L})$ & $42.9 \pm 1.7$ & $49.7 \pm 2.6$ & $0.044^{*}$ \\
EDV $(\mu \mathrm{L})$ & $55.1 \pm 2.4$ & $59.8 \pm 1.7$ & 0.15 \\
ESP $(\mathrm{mmHg})$ & $114 \pm 3$ & $102 \pm 4$ & $0.031^{*}$ \\
EDP $(\mathrm{mmHg})$ & $7.5 \pm 1.0$ & $9.1 \pm 2.3$ & 0.566 \\
$\mathrm{dP} / \mathrm{dt}$ max $(\mathrm{mmHg} / \mathrm{s})$ & $9377 \pm 707$ & $6532 \pm 759$ & $0.019^{*}$ \\
$\mathrm{dP} / \mathrm{dt}$ min $(\mathrm{mmHg} / \mathrm{s})$ & $-6932 \pm 243$ & $-6197 \pm 600$ & 0.296 \\
PRSW & $54.3 \pm 6.1$ & $30.3 \pm 6.4$ & $0.020^{*}$ \\
Ees & $6.96 \pm 1.64$ & $1.81 \pm 0.59$ & $0.020^{*}$ \\
EDPVR slope & $0.41 \pm 0.29$ & $4.49 \pm 3.97$ & 0.352 \\
Tau (ms) & $8.87 \pm 0.25$ & $10.27 \pm 1.10$ & 0.268 \\
Heart rate (bpm) & $435 \pm 17$ & $433 \pm 16$ & 0.948 \\
Body weight & $21.2 \pm 1.8$ & $22.4 \pm 1.8$ & 0.272 \\
\hline
\end{tabular}

ESV, end-systolic volume; EDV, end-diastolic volume; ESP, end-systolic pressure; $E D P$, end-diastolic pressure; $d P / d t$ max, peak rate of pressure rise; $d P / d t$ min, peak rate of pressure decline; PRSW, preload recruited stroke work; Ees, endsystolic elastance; EDPVR slope, end-diastolic PV relation slope; Tau, relaxation time constant. $P, P$ value. ${ }^{*} P<0.05$.

mice at time 0 , but was not significantly changed within $48 \mathrm{~h}$ with or without UPS inhibitors (Figures 4A,B). Thus, UPS-dependent protein degradation may not be responsible for the reduction of cMLCK proteins in heterozygous knockout cardiomyocytes.

Despite the marked reduction of cMLCK proteins in heterozygous knockout relative to control hearts, intracellular localization of cMLCK in hearts was similar, showing a striated pattern with additional diffuse staining in the cytoplasm (Figure 4C, green). Striated cMLCK staining was not colocalized with MLC2v (Figure 4C, red) both in wild-type as well as heterozygous knockout hearts, which is consistent with our previous study (Chan et al., 2008). Co-immunostaining of cMLCK and Z-disk protein $\alpha$-actinin 2 revealed overlapping localization, while co-immunostaining of cMLCK and M-line protein myomesin did not in mouse hearts (Figure 4D).

\section{DISCUSSION}

Reduced phosphorylation of MLC2v, a process primarily regulated by cMLCK, has been implicated in human heart disease (Morano, 1999; van der Velden et al., 2003). Recent studies

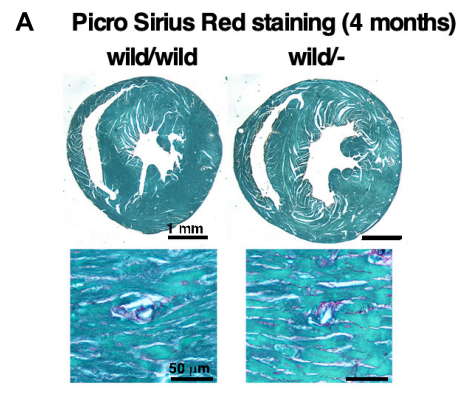

B 4 months isolated myocytes

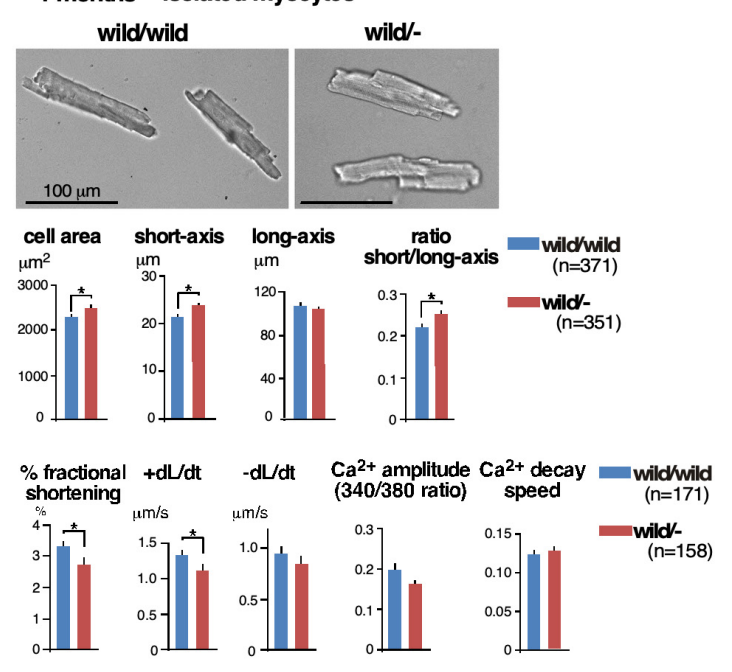

FIGURE 2 | Enlarged cardiomyocytes with reduced contractility and impaired $\mathrm{Ca}^{2+}$-handling in heterozygous Mylk3 knockout mice. (A) Representative images of Picro Sirius Red-stained transverse sections of the hearts.

(B) Representative images of cardiomyocytes, short axis $(\mu \mathrm{m})$, long axis $(\mu \mathrm{m})$, cell area $\left(\mu \mathrm{m}^{2}\right)$ and ratio of short vs. long axis of cardiomyocytes isolated at 4 months of age (Mylk3 $3^{\text {wild/wild }}, n=371 ;$ Mylk3 $3^{\text {wild } /-}, n=351$ from $n=3$ mice each). Bars $=100 \mu \mathrm{m}$. (C) Measurements of cardiac contraction and simultaneous $\mathrm{Ca}^{2+}$ transients in isolated cardiomyocytes (Mylk3 wild/wild, $n=171 ;$ Mylk3 wild $/-, n=158$ from $n=3$ mice each). Data are expressed as mean \pm SEM. ${ }^{*} P<0.05$. $+\mathrm{dL} / \mathrm{dt}$ (speed of contraction); $-\mathrm{dL} / \mathrm{dt}$ (speed of relaxation).

have proposed a novel role for cMLCK in the pathogenesis of DCM following the identification of heterozygous variants in the MYLK3 gene encoding cMLCK in individuals with disease (Tobita et al., 2017; Hodatsu et al., 2019). To consider the possibility that these heterozygous variants could be loss-of-function mutations (haploinsufficiency), we analyzed heterozygous $M y l k 3$ knockout mice for the first time.

The young mice at 4 months of age showed mild cardiac dysfunction with increased cardiomyocyte area size and shortaxis length relative to the cardiomyocytes from control mice. Expression of cMLCK mRNA was expectedly reduced by almost half, however, protein expression was further reduced in heterozygous knockout relative to wild-type mice. Notably, it is often found that heterozygous knockout mice express more or less than half of the proteins relative to control wild-type mice in other genes (Gineste et al., 2013; Zhou et al., 2015; 
A 4 months - protein expression

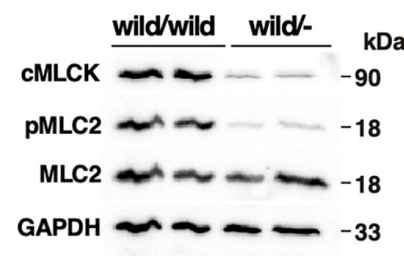

B CMLCKJGAPDH pMLC2/total MLC2

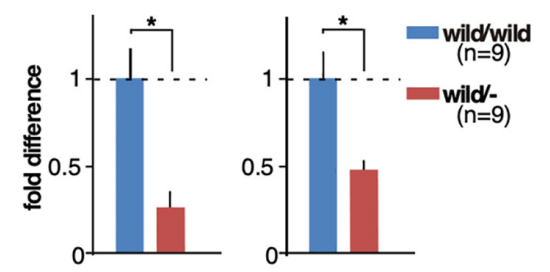

C 4 months - mRNA
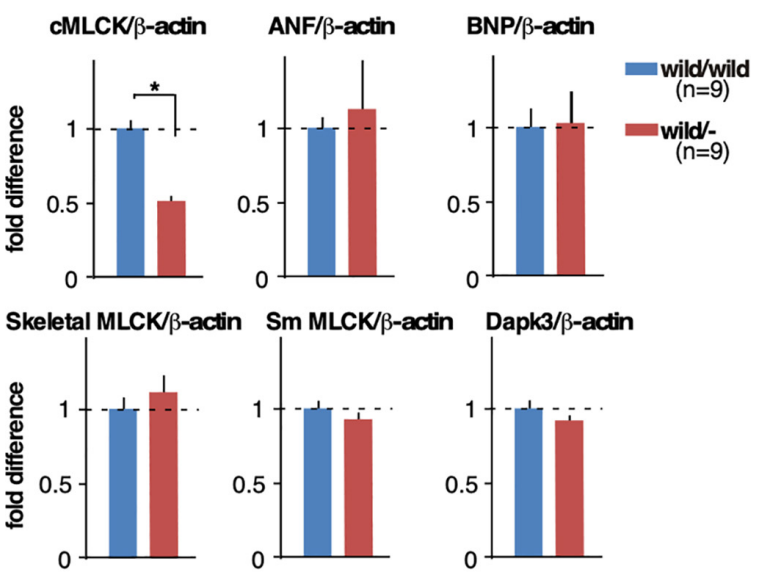

FIGURE 3 | Reduced cMLCK proteins and MLC2 phosphorylation in heterozygous Mylk3 knockout hearts. (A) Representative Western blotting of cMLCK, pMLC2v, total MLC2v and GAPDH using isolated hearts from Mylk3 ${ }^{\text {wild/wild }}$ and Mylk3 ${ }^{\text {wild/ }}-$ mice at 4 months of age. The cropped blots are used in the figure. (B) Fold difference of cMLCK, pMLC2v and total MLC2v normalized to GAPDH with the value of Mylk3wild/wild defined as 1 ( $n=9$ each). (C) Real-time RT-PCR demonstrates relative expression of cMLCK, atrial natriuretic factor (ANF), brain natriuretic peptide (BNP), skeletal MLCK, smooth muscle MLCK, and Dapk3 mRNA normalized to $\beta$-actin with the value in Mylk3 ${ }^{\text {wild/wild }}$ defined as 1 . Data are expressed as mean \pm SEM. ${ }^{*} P<0.05$. ANF, atrial natriuretic factor; BNP, brain natriuretic peptide; Sm, smooth muscle.

Zhang et al., 2017). An approximate $75 \%$ reduction in cMLCK proteins and a consequent reduction in MLC2 phosphorylation by approximately half may lead to reduced contractility if the observation in human failing hearts is applied to mice. An approximate 50\% reduction in MLC2 phosphorylation was found in human failing hearts (Morano, 1999; van der Velden et al., 2003). In normal wild-type mice, two serine residues positioning at 15 and 16 in MLC2v are phosphorylated (Sheikh et al., 2012). When these two serine residues were mutated into alanine in homozygous knock-in mice, these mice developed the DCM phenotype accompanied with loss of MLC2v phosphorylation (Sheikh et al., 2012). Whether heterozygous knock-in mice develop heart failure remains to be studied, which will help us to understand whether haploinsufficiency of the Mylk3 gene or a reduction in MLC2 phosphorylation is the primary or secondary trigger of cardiac dysfunction observed in heterozygous Mylk3 knockout mice.

The phenotype demonstrated in heterozygous Mylk3 knockout mice is milder than the reported phenotype seen in humans with heterozygous variants in the MYLK3 gene. There are several possible explanations for the phenotypic difference between heterozygous variants in human and heterozygous knockout in mice. It could be due to species effects, in other words mouse as opposed to man (Hacking, 2008). A second possibility is that the effect of human variants may differ from the effect of the haploinsufficiency resulting from heterozygous knockout. cMLCK proteins are composed of roughly three different domains: the amino terminal domain unique to the cardiac isoform without homologies to other MLCKs such as smooth muscle or skeletal types, followed by the conserved catalytic and regulatory domains (Seguchi et al., 2007; Chan et al., 2008). To our knowledge, three different mutations in MYLK3 gene have been identified (Tobita et al., 2017; Hodatsu et al., 2019). Two mutations are predicted to generate a shorter protein with disruption of the catalytic and regulatory domains. Another mutation at the stop codon is predicted to generate a longer protein with an additional 19 amino acids at the carboxyl-terminus. It might be anticipated that for such diverse effects, such as haploinsufficiency compared with dominant-negative, there would be associated variation in clinical phenotypes (Kasahara and Benson, 2004). A third possibility is that patients with heterozygous mutations in MYLK3 gene may carry additional genetic mutations in other genes that may contribute to the phenotypic presentation. In fact, an additional nonsense mutation in a filamin C (FLNC) gene was found in some of the family members exhibiting early onset of DCM (Tobita et al., 2017), and genetic linkages between the FLNC gene and DCM have been reported (Ortiz-Genga et al., 2016; Reinstein et al., 2016). 

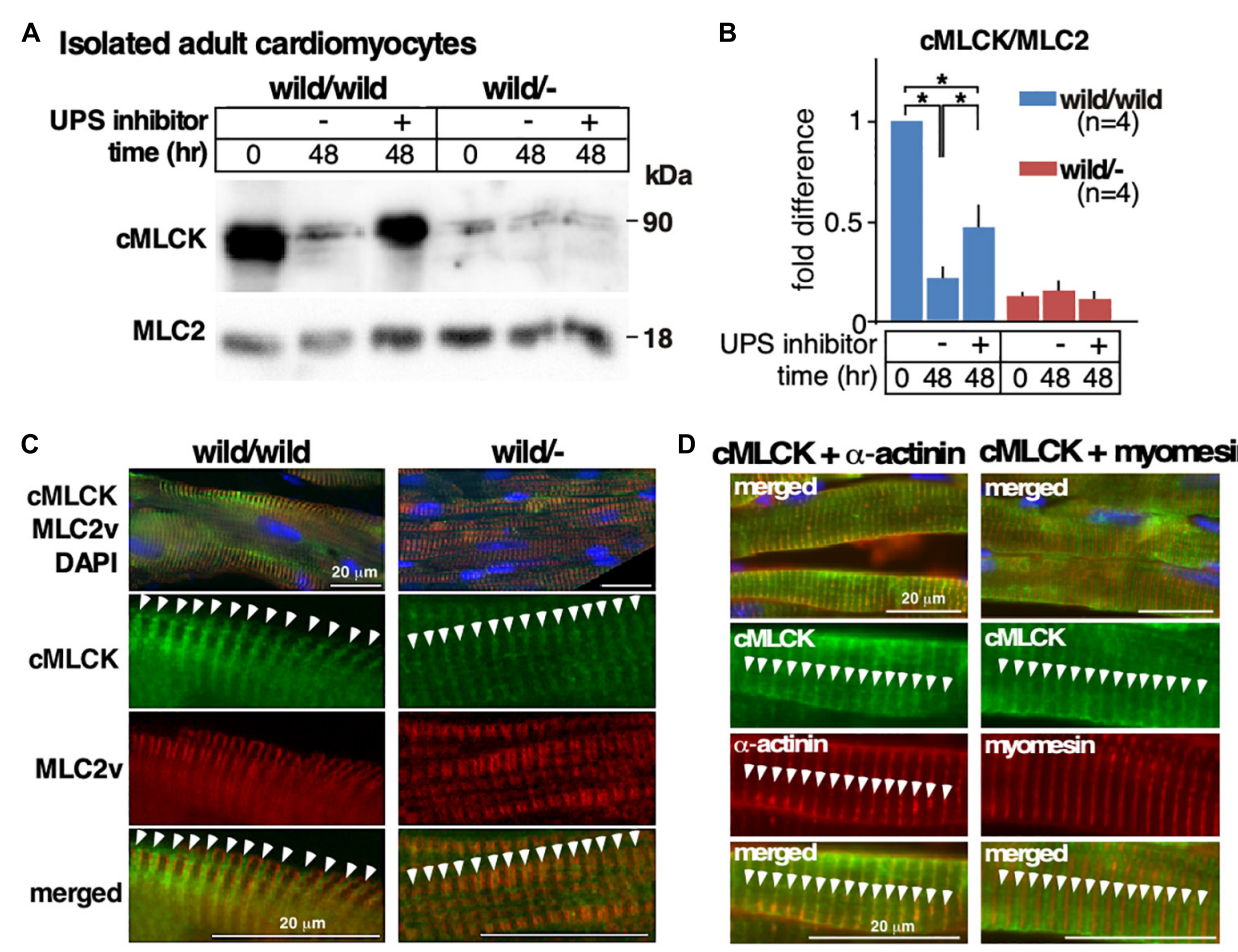

FIGURE 4 | Altered responses to UPS inhibitors and cycloheximide between heterozygous knockout and wild-type adult cardiomyocytes. (A) Representative Western blotting of cMLCK and total MLC2v using adult cardiomyocytes isolated from Mylk3 wild/wild and Mylk3wild/- mice at 4 months of age at time 0 and 48 h of incubation with or without UPS inhibitors. The cropped blots are used in the figure. (B) Fold difference of cMLCK relative to total MLC2v with the value of Mylk3 $3^{\text {wild/wild }}$ at time 0 defined as 1 ( $n=4$ each). (C) Representative immunostaining of cMLCK and MLC2v in the heart sections ( $n=3$ each). (D) Representative immunostaining of cMLCK and Z-disk protein $\alpha$-actinin2, and CMLCK and M-line protein myomesin in the heart sections. Data are expressed as mean \pm SEM. $* P<0.05$. Bars $=20 \mu \mathrm{m}$.

Despite that one allele in the human MYLK3 gene being intact in these patients, cMLCK protein expression in patients' hearts was reported to be markedly reduced compared to control (Tobita et al., 2017). In heterozygous Mylk3 knockout hearts, we also observed a marked reduction of cMLCK proteins relative to the wild-type mice. We attempted to compare the differences in UPS-dependent protein degradation between heterozygous knockout and controls using isolated adult cardiomyocytes. In the wild-type cardiomyocytes cMLCK proteins were markedly downregulated within $48 \mathrm{~h}$ of culture, and this was partly blocked by UPS inhibitors. On the other hand, expression of cMLCK in heterozygous knockout cardiomyocytes was lower than wildtype cardiomyocytes shortly after isolation from hearts, but was not significantly changed within $48 \mathrm{~h}$ of culture regardless of UPS inhibitor presence. These results indicate that expression of cMLCK protein in hearts and isolated cardiomyocytes is regulated by its environment, namely, the dynamic environment of contractile hearts with over 600 beats per minute in vivo versus the static environment of isolated cardiomyocytes. In addition, there were distinct differences between wild-type and heterozygous knockout cardiomyocytes regarding the abundance of cMLCK proteins during $48 \mathrm{~h}$ of culture and responses to UPS inhibitors; however, our hypothesis regarding the increase of UPS-dependent protein degradation in heterozygous knockout cardiomyocytes relative to control was not supported.
The intracellular localization of the cMLCK protein was similar between control and heterozygous cMLCK knockout mice, showing a striated pattern co-localized not with MLC2v, but with Z-disk protein alpha-actinin, with additional diffuse staining in the cytoplasm. Studies into whether this intracellular localization has additional roles in cMLCK are currently underway.

In summary, we report that the heterozygous lossof-function mutation of Mylk3 in mice shows a mild reduction in cardiac contractility and a reduction in proteins by approximately $75 \%$ relative to the control wild-type mice. These phenotypes partly represent DCM in humans with the heterozygous MYLK3 mutation, but are milder. This animal model has the potential to shed light on understanding the mechanisms of low cMLCK protein expression in human DCM patients with heterozygous MYLK3 mutations.

\section{ETHICS STATEMENT}

This study was carried out in accordance with the recommendations of the $\mathrm{NIH}$ guidelines. The protocol was approved by University of Florida Institutional Animal Care and Use Committee. 


\section{AUTHOR CONTRIBUTIONS}

All authors designed and performed the experiments. CT and HK prepared the manuscript.

\section{FUNDING}

This work was supported by the American Heart Association (17GRNT33411076 to HK) and University of Florida MSRP Scholars Program (T35 HL007489 to CT).

\section{REFERENCES}

Bartha, I., di Iulio, J., Venter, J. C., and Telenti, A. (2018). Human gene essentiality. Nat. Rev. Genet. 19, 51-62. doi: 10.1038/nrg.2017.75

Chan, J. Y., Takeda, M., Briggs, L. E., Graham, M. L., Lu, J. T., Horikoshi, N., et al. (2008). Identification of cardiac-specific myosin light chain kinase. Circ. Res. 102, 571-580. doi: 10.1161/CIRCRESAHA.107.161687

Chang, A. N., Chen, G., Gerard, R. D., Kamm, K. E., and Stull, J. T. (2010). Cardiac myosin is a substrate for zipper-interacting protein kinase (ZIPK). J. Biol. Chem. 285, 5122-5126. doi: 10.1074/jbc.C109.076489

Davis, J. S., Hassanzadeh, S., Winitsky, S., Lin, H., Satorius, C., Vemuri, R., et al. (2001). The overall pattern of cardiac contraction depends on a spatial gradient of myosin regulatory light chain phosphorylation. Cell 107, 631-641.

Gineste, C., De Winter, J. M., Kohl, C., Witt, C. C., Giannesini, B., Brohm, K., et al. (2013). In vivo and in vitro investigations of heterozygous nebulin knockout mice disclose a mild skeletal muscle phenotype. Neuromuscul. Disord. 23, 357-369. doi: 10.1016/j.nmd.2012.12.011

Hacking, D. F. (2008). 'Knock, and it shall be opened': knocking out and knocking in to reveal mechanisms of disease and novel therapies. Early Hum. Dev. 84, 821-827. doi: 10.1016/j.earlhumdev.2008.09.011

Herring, B. P., Dixon, S., and Gallagher, P. J. (2000). Smooth muscle myosin light chain kinase expression in cardiac and skeletal muscle. Am. J. Physiol. Cell Physiol. 279, C1656-C1664.

Hodatsu, A., Fujino, N., Uyama, Y., Tsukamoto, O., Imai-Okazaki, A., Yamazaki, S., et al. (2019). Impact of cardiac myosin light chain kinase gene mutation on development of dilated cardiomyopathy. ESC Heart Fail. 6, 406-415. doi: 10.1002/ehf2.12410

Kasahara, H., and Benson, D. W. (2004). Biochemical analyses of eight NKX2.5 homeodomain missense mutations causing atrioventricular block and cardiac anomalies. Cardiovasc. Res. 64, 40-51.

Marston, S., Copeland, O., Gehmlich, K., Schlossarek, S., and Carrier, L. (2012). How do MYBPC3 mutations cause hypertrophic cardiomyopathy? J. Muscle Res. Cell Motil. 33, 75-80. doi: 10.1007/s10974-011-9268-3

Massengill, M. T., Ashraf, H. M., Chowdhury, R. R., Chrzanowski, S. M., Kar, J., Warren, S. A., et al. (2016). Acute heart failure with cardiomyocyte atrophy induced in adult mice by ablation of cardiac myosin light chain kinase. Cardiovasc. Res. 111, 34-43. doi: 10.1093/cvr/cvw069

Morano, I. (1999). Tuning the human heart molecular motors by myosin light chains. J. Mol. Med. 77, 544-555.

Moss, R. L., and Fitzsimons, D. P. (2006). Myosin light chain 2 into the mainstream of cardiac development and contractility. Circ. Res. 99, 225-227.

Ortiz-Genga, M. F., Cuenca, S., Dal Ferro, M., Zorio, E., Salgado-Aranda, R., Climent, V., et al. (2016). Truncating FLNC mutations are associated with highrisk dilated and arrhythmogenic cardiomyopathies. J. Am. Coll. Cardiol. 68, 2440-2451. doi: 10.1016/j.jacc.2016.09.927

Pacher, P., Nagayama, T., Mukhopadhyay, P., Bátkai, S., and Kass, D. A. (2008). Measurement of cardiac function using pressure-volume conductance catheter technique in mice and rats. Nat. Protoc. 3, 1422-1434. doi: 10.1038/nprot. 2008.138

Reinstein, E., Gutierrez-Fernandez, A., Tzur, S., Bormans, C., Marcu, S., TayebFligelman, E., et al. (2016). Congenital dilated cardiomyopathy caused by biallelic mutations in Filamin C. Eur. J. Hum. Genet. 24, 1792-1796. doi: 10. 1038/ejhg.2016.110

\section{ACKNOWLEDGMENTS}

We are grateful to N. Terada, E. Chan, J. Calise, C. Lacko, and C. Astrom for valuable suggestions and technical supports.

\section{SUPPLEMENTARY MATERIAL}

The Supplementary Material for this article can be found online at: https://www.frontiersin.org/articles/10.3389/fphys. 2019.00696/full\#supplementary-material

Rocha, H., Sampaio, M., Rocha, R., Fernandes, S., and Leão, M. (2016). MEF2C haploinsufficiency syndrome: report of a new MEF2C mutation and review. Eur. J. Med. Genet. 59, 478-482. doi: 10.1016/j.ejmg.2016.05.017

Sanbe, A., Fewell, J. G., Gulick, J., Osinska, H., Lorenz, J., Hall, D. G., et al. (1999). Abnormal cardiac structure and function in mice expressing nonphosphorylatable cardiac regulatory myosin light chain 2. J. Biol. Chem. 274, 21085-21094.

Scruggs, S. B., and Solaro, R. J. (2011). The significance of regulatory light chain phosphorylation in cardiac physiology. Arch. Biochem. Biophys. 510, 129-134. doi: 10.1016/j.abb.2011.02.013

Seguchi, O., Takashima, S., Yamazaki, S., Asakura, M., Asano, Y., Shintani, Y., et al. (2007). A cardiac myosin light chain kinase regulates sarcomere assembly in the vertebrate heart. J. Clin. Invest. 117, 2812-2824.

Sheikh, F., Ouyang, K., Campbell, S. G., Lyon, R. C., Chuang, J., Fitzsimons, D., et al. (2012). Mouse and computational models link Mlc2v dephosphorylation to altered myosin kinetics in early cardiac disease. J. Clin. Invest. 122, 12091221. doi: 10.1172/JCI61134

Stelzer, J. E., Patel, J. R., and Moss, R. L. (2006). Acceleration of stretch activation in murine myocardium due to phosphorylation of myosin regulatory light chain. J. Gen. Physiol. 128, 261-272.

Takeda, M., Briggs, L. E., Wakimoto, H., Marks, M. H., Warren, S. A., Lu, J. T., et al. (2009). Slow progressive conduction and contraction defects in loss of Nkx25 mice after cardiomyocyte terminal differentiation. Lab. Invest. 89, 983-993. doi: 10.1038/labinvest.2009.59

Tobita, T., Nomura, S., Morita, H., Ko, T., Fujita, T., Toko, H., et al. (2017). Identification of MYLK3 mutations in familial dilated cardiomyopathy. Sci. Rep. 7:17495. doi: 10.1038/s41598-017-17769-1

van der Velden, J., Papp, Z., Boontje, N. M., Zaremba, R., de Jong, J. W., Janssen, P. M. L., et al. (2003). The effect of myosin light chain 2 dephosphorylation on $\mathrm{Ca} 2+-$ sensitivity of force is enhanced in failing human hearts. Cardiovasc. Res. 57, 505-514.

Warren, S. A., Briggs, L. E., Zeng, H., Chuang, J., Chang, E. I., Terada, R., et al. (2012). Myosin light chain phosphorylation is critical for adaptation to cardiac stress. Circulation 126, 2575-2588. doi: 10.1161/CIRCULATIONAHA. 112.116202

Zhang, Y., Qian, Y., Zhang, J., Yan, W., Jung, Y. S., Chen, M., et al. (2017). Ferredoxin reductase is critical for p53-dependent tumor suppression via iron regulatory protein 2. Genes Dev. 31, 1243-1256. doi: 10.1101/gad.299388.117

Zhou, C., Ding, L., Deel, M. E., Ferrick, E. A., Emeson, R. B., Gallagher, M. J., et al. (2015). Altered intrathalamic GABAA neurotransmission in a mouse model of a human genetic absence epilepsy syndrome. Neurobiol. Dis. 73, 407-417. doi: $10.1016 /$ j.nbd.2014.10.021

Conflict of Interest Statement: The authors declare that the research was conducted in the absence of any commercial or financial relationships that could be construed as a potential conflict of interest.

Copyright (c) 2019 Tougas, Grindrod, Cai, Alkassis and Kasahara. This is an openaccess article distributed under the terms of the Creative Commons Attribution License (CC BY). The use, distribution or reproduction in other forums is permitted, provided the original author(s) and the copyright owner(s) are credited and that the original publication in this journal is cited, in accordance with accepted academic practice. No use, distribution or reproduction is permitted which does not comply with these terms. 